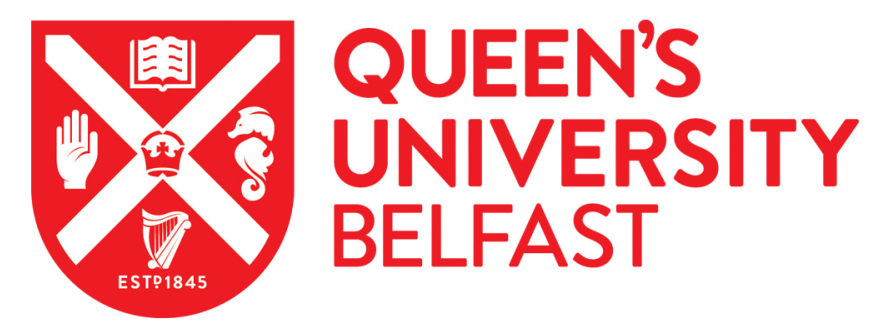

\title{
Performance of African-ancestry-specific polygenic hazard score varies according to local ancestry in 8q24
}

UKGPCS collaborators (2021). Performance of African-ancestry-specific polygenic hazard score varies according to local ancestry in 8q24. Prostate Cancer and Prostatic Diseases. https://doi.org/10.1038/s41391021-00403-7

Published in:

Prostate Cancer and Prostatic Diseases

Document Version:

Peer reviewed version

Queen's University Belfast - Research Portal:

Link to publication record in Queen's University Belfast Research Portal

Publisher rights

Copyright 2021, the Authors.

This work is made available online in accordance with the publisher's policies. Please refer to any applicable terms of use of the publisher.

\section{General rights}

Copyright for the publications made accessible via the Queen's University Belfast Research Portal is retained by the author(s) and / or other copyright owners and it is a condition of accessing these publications that users recognise and abide by the legal requirements associated with these rights.

Take down policy

The Research Portal is Queen's institutional repository that provides access to Queen's research output. Every effort has been made to ensure that content in the Research Portal does not infringe any person's rights, or applicable UK laws. If you discover content in the Research Portal that you believe breaches copyright or violates any law, please contact openaccess@qub.ac.uk. 


\section{Performance of African-ancestry-specific polygenic hazard score varies according to local ancestry in $8 q 24$}

Roshan A. Karunamuni ${ }^{1}$, Minh-Phuong Huynh-Le², Chun C. Fan ${ }^{3}$, Wesley Thompson4, Asona

5 Lui ${ }^{1}$, Maria Elena Martinez ${ }^{5,6}$, Brent S. Rose ${ }^{1}$, Brandon Mahal $^{7}$, Rosalind A. Eeles ${ }^{8,9}$, Zsofia Kote-Jarai ${ }^{8}$, Kenneth Muir ${ }^{10,11}$, Artitaya Lophatananon ${ }^{10}$, UKGPCS collaborators ${ }^{12}$, Catherine M.

Tangen $^{13}$, Phyllis J. Goodman ${ }^{13}$, lan M. Thompson Jr. ${ }^{14}$, William J. Blot ${ }^{15,16}$, Wei Zheng ${ }^{15}$, Adam

S. Kibel ${ }^{17}$, Bettina F. Drake ${ }^{18}$, Olivier Cussenot ${ }^{19,20}$, Géraldine Cancel-Tassin ${ }^{20,19}$, Florence Menegaux ${ }^{21}$, Thérèse Truong ${ }^{21}$, Jong Y. Park22, Hui-Yi Lin ${ }^{23}$, Jack A. Taylor ${ }^{24,25}$, Jeannette T.

10 Bensen ${ }^{26,27}$, James L. Mohler ${ }^{28,27}$, Elizabeth T.H. Fontham ${ }^{29}$, Luc Multigner ${ }^{30}$, Pascal Blanchet ${ }^{31}$, Laurent Brureau ${ }^{31}$, Marc Romana ${ }^{32,33}$, Robin J. Leach ${ }^{34}$, Esther M. John ${ }^{35}$, Jay H. Fowke ${ }^{36,37}$, William S. Bush ${ }^{38}$, Melinda C. Aldrich ${ }^{39}$, Dana C. Crawford ${ }^{40}$, Jennifer Cullen ${ }^{41}$, Gyorgy Petrovics ${ }^{42}$, Marie-Élise Parent ${ }^{43,44}$, Jennifer J. $\mathrm{Hu}^{45}$, Maureen Sanderson ${ }^{46}$, The PRACTICAL Consortium ${ }^{47}$, Ian G. Mills ${ }^{48}$, Ole A. Andreassen ${ }^{49}$, Anders M. Dale ${ }^{50}$, Tyler M. Seibert ${ }^{1,50,51}$

15 1Department of Radiation Medicine and Applied Sciences, University of California San Diego, La Jolla, CA, USA

${ }^{2}$ Radiation Oncology, George Washington University, Washington, DC

${ }^{3}$ Center for Human Development, University of California San Diego, La Jolla, CA, USA

${ }^{4}$ Department of Family Medicine and Public Health, University of California, San Diego, La Jolla,

20 CA, USA

${ }^{5}$ Herbert Wertheim School of Public Health and Human Longevity Science, University of California San Diego, La Jolla, California

${ }^{6}$ Moores Cancer Center, University of California San Diego, La Jolla, California

${ }^{7}$ Department of Radiation Oncology, University of Miami Miller School of Medicine, Miami, FL

25 8The Institute of Cancer Research, London, SM2 5NG, UK ${ }^{9}$ Royal Marsden NHS Foundation Trust, London, SW3 6JJ, UK ${ }^{10}$ Division of Population Health, Health Services Research and Primary Care, University of Manchester, Oxford Road, Manchester, M13 9PL, UK ${ }^{11}$ Warwick Medical School, University of Warwick, Coventry, CV4 7AL, UK

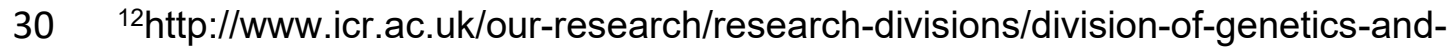
epidemiology/oncogenetics/research-projects/ukgpcs/ukgpcs-collaborators ${ }^{13}$ SWOG Statistical Center, Fred Hutchinson Cancer Research Center, Seattle, WA, USA ${ }^{14}$ CHRISTUS Santa Rosa Hospital - Medical Center, San Antonio, TX, USA

${ }^{15}$ Division of Epidemiology, Department of Medicine, Vanderbilt University Medical Center, 2525 
West End Avenue, Suite 800, Nashville, TN 37232 USA.

${ }^{16}$ International Epidemiology Institute, Rockville, MD 20850, USA

${ }^{17}$ Division of Urologic Surgery, Brigham and Womens Hospital, 75 Francis Street, Boston, MA 02115, USA

5 18Washington University School of Medicine, 660 S. Euclid Avenue, Campus Box 8242, St. Louis, MO 63110 , USA

${ }^{19}$ Sorbonne Universite, GRC n5, AP-HP, Tenon Hospital, 4 rue de la Chine, F-75020 Paris, France ${ }^{20} \mathrm{CeRePP}$, Tenon Hospital, F-75020 Paris, France.

$10{ }^{21}$ CESP (UMR 1018), Faculté de Médecine, Université Paris-Saclay, Inserm, Gustave Roussy, Villejuif, France

${ }^{22}$ Department of Cancer Epidemiology, Moffitt Cancer Center, 12902 Magnolia Drive, Tampa, FL 33612, USA

${ }^{23}$ School of Public Health, Louisiana State University Health Sciences Center, New Orleans, LA

15 70112, USA

${ }^{24}$ Epidemiology Branch, National Institute of Environmental Health Sciences, Research Triangle Park, NC, USA

${ }^{25}$ Epigenetic and Stem Cell Biology Laboratory, National Institute of Environmental Health Sciences, Research Triangle Park, NC

$20{ }^{26}$ Department of Epidemiology, University of North Carolina at Chapel Hill, Chapel Hill, NC, USA ${ }^{27}$ Lineberger Comprehensive Cancer Center, University of North Carolina at Chapel Hill, Chapel Hill, NC, USA

${ }^{28}$ Department of Urology, Roswell Park Comprehensive Cancer Center, Buffalo, NY, USA ${ }^{29}$ School of Public Health, Louisiana State University Health Sciences Center, New Orleans, LA,

25 USA

30Univ Rennes, Inserm, EHESP, Irset (Institut de recherche en santé, environnement et travail) UMR_S 1085, Rennes, France

${ }^{31} \mathrm{CHU}$ de Pointe-à-Pitre, Univ Antilles, Univ Rennes, Inserm, EHESP, Irset (Institut de recherche en santé, environnement et travail) - UMR_S 1085, Pointe-à-Pitre, France

30 32UMR Inserm 1134, Biologie Intégrée du Globule Rouge, INSERM/Université Paris DiderotUniversité Sorbonne Paris Cité/INTS/Université des Antilles, Guadeloupe, France ${ }^{33}$ Laboratoire d'Excellence GR-Ex « The red cell: from genesis to death », PRES Sorbonne Paris Cité, Paris, France

${ }^{34}$ Department of Cell Systems and Anatomy, Mays Cancer Center, University of Texas Health

35 Science Center at San Antonio, San Antonio Texas

${ }^{35}$ Departments of Epidemiology \& Population Health and of Medicine, Division of Oncology, Stanford Cancer Institute, Stanford University School of Medicine, Stanford, CA 94304 USA ${ }^{36}$ Department of Medicine and Urologic Surgery, Vanderbilt University Medical Center, 1211 Medical Center Drive, Nashville, TN 37232, USA

$40 \quad{ }^{37}$ Division of Epidemiology, Department of Preventive Medicine, The University of Tennessee Health Science Center, TN, USA

${ }^{38}$ Case Western Reserve University, Department of Population and Quantitative Health Sciences, Cleveland Institute for Computational Biology, 2103 Cornell Road, Wolstein Research Building, Suite 2530, Cleveland, OH, 44106 USA 
${ }^{39}$ Vanderbilt University Medical Center, Division of Genetic Medicine, Department of Medicine, 519A Light Hall, 2215 Garland Avenue, Nashville, TN 37232-4682 USA

${ }^{40}$ Case Western Reserve University, Department of Population and Quantitative Health Sciences, Cleveland Institute for Computational Biology, 2103 Cornell Road, Wolstein Research

$5 \quad$ Building, Suite 2527, Cleveland, OH, 44106 USA

${ }^{41}$ Department of Population and Quantitative Health Sciences, Case Western Reserve University, Cleveland, OH 44106-7219, USA

${ }^{42}$ Center for Prostate Disease Research, 6720A Rockledge Drive, Suite 300, Bethesda, MD 20817, USA

$10 \quad{ }^{43}$ Epidemiology and Biostatistics Unit, Centre Armand-Frappier Santé Biotechnologie, Institut national de la recherche scientifique, 531 Boul. des Prairies, Laval, QC, Canada H7V 1B7 ${ }^{44}$ Department of Social and Preventive Medicine, School of Public Health, University of Montreal, Montreal, QC, Canada

${ }^{45}$ The University of Miami School of Medicine, Sylvester Comprehensive Cancer Center, 1120

15 NW 14th Street, CRB 1511, Miami, Florida 33136, USA

${ }^{46}$ Department of Family and Community Medicine, Meharry Medical College, 1005 Dr. DB Todd Jr. Blvd., Nashville, TN 37208 USA

${ }^{47}$ Institute of Cancer Research, Sutton, SW7 3RPm UK

${ }^{48}$ Center for Cancer Research and Cell Biology, Queen's University of Belfast, Belfast, UK

$20{ }^{49}$ NORMENT, KG Jebsen Centre, Oslo University Hospital and University of Oslo, Oslo, Norway

${ }^{50}$ Department of Radiology, University of California San Diego, La Jolla, CA, USA

${ }^{51}$ Department of Bioengineering, University of California San Diego, La Jolla, CA, USA

25 * Additional consortia memebers, associated funding, and contact information are provided in the Supplementary Data Appendix 1,2, and 3.

\section{Corresponding Author:}

Roshan Karunamuni, PhD

30 University of California, San Diego

Department of Radiation Medicine and Applied Sciences

3960 Health Sciences Dr, Mail Code 0865

La Jolla, CA 92093

rakarunamuni@health.ucsd.edu

35 ORCID: 0000-0001-8723-8123

Tyler M. Seibert, MD, PhD

University of California, San Diego

Department of Radiation Medicine and Applied Sciences 
La Jolla, CA 92093

tseibert@health.ucsd.edu

ORCID: 0000-0002-4089-7399

$5 \quad$ Running title

Performance of polygenic score varies with $8 q 24$ ancestry

\section{Keywords}

Prostate cancer; health disparities; genome wide association study; polygenic risk; genomics;

10 local genotypic ancestry; African

\section{Funding}

This study was funded in part by grants from the University of California (\#C21CR2060), the United States National Institute of Health/National Institute of Biomedical Imaging and

15 Bioengineering (\#K08EB026503), the Research Council of Norway (\#223273), KG Jebsen Stiftelsen, and South East Norway Health Authority.

Preprint DOI: https://www.medrxiv.org/content/10.1101/2021.01.20.21249985v1 


\section{Abstract}

Background: We previously developed an African-ancestry-specific polygenic hazard score (PHS46+African) that substantially improved prostate cancer risk stratification in men with African ancestry. The model consists of 46 SNPs identified in Europeans and 3 SNPs from 8q24

5 shown to improve model performance in Africans. Herein, we used principal component (PC) analysis to uncover subpopulations of men with African ancestry for whom the utility of PHS46+African may differ.

Materials and Methods: Genotypic data were obtained from PRACTICAL consortium for 6,253 men with African genetic ancestry. Genetic variation in a window spanning 3 African-specific

10 8q24 SNPs was estimated using 93 PCs. A Cox proportional hazards framework was used to identify the pair of PCs most strongly associated with performance of PHS46+African. A calibration factor (CF) was formulated using Cox coefficients to quantify the extent to which the performance of PHS46+African varies with PC.

Results: CF of PHS46+African was strongly associated with the first and twentieth PCs.

15 Predicted CF ranged from 0.41 to 2.94 , suggesting that PHS46+African may be up to 7 times more beneficial to some African men than others. The explained relative risk for PHS46+African varied from $3.6 \%$ to $9.9 \%$ for individuals with low and high CF values, respectively. By crossreferencing our dataset with 1000 Genomes, we identified significant associations between continental and calibration groupings.

20 Conclusion: We identified PCs within 8q24 that were strongly associated with performance of PHS46+African. Further research to improve clinical utility of polygenic risk scores (or models) is needed to improve health outcomes for men of African ancestry 


\section{Introduction}

Polygenic hazard score (PHS) models can test for associations between genetic variants and the age at diagnosis of prostate cancer ${ }^{1,2}$. These models generate personalized estimates of risk that can be used to guide decisions on whether and when to offer screening to men ${ }^{3-5}$.

5 However, development of polygenic models have often included only individuals of European genetic ancestry ${ }^{6,7}$, which could potentially lead to greater prostate cancer disparities. This is a particular concern for prostate cancer. For example, African American men are more likely than other men in the United States to develop prostate cancer, have a younger age of diagnosis, and are more than twice as likely to die from their prostate cancer as white men ${ }^{8}$.

PHS46, a model for prostate cancer trained exclusively in men of European ancestry, was found to be roughly half as effective in African men as in Europeans and Asians ${ }^{9}$. Similar trends were observed for other polygenic scores ${ }^{6}$, highlighting the need for increased diversification of large-scale genome studies in order to address this disparity ${ }^{7}$ in clinical utility. Furthermore, studies have suggested that inequities in the performance of polygenic risk scores

15 may exacerbate disparities for individuals and communities that are already under-represented in research ${ }^{10}$. In an effort to develop more equitable PHS models, our group recently developed an African-ancestry-specific PHS model (PHS46+African) ${ }^{11}$, that substantially improved upon the performance of PHS46.

PHS46+African consists of 46 single nucleotide polymorphisms (SNPs) that were

20 identified in Europeans, together with 3 additional SNPs located on the 8q24 chromosome. These three SNPs, herein referred to as African-specific, were found to uniquely improve performance of the PHS model in men of African genetic ancestry (African men). The term "African-specific" is not meant to confer any information on the relative allele frequency of these variants. However, given the inherent genetic diversity on the African continent and gene flow

25 associated with the African diaspora ${ }^{12}$, we believe that PHS46+African may benefit certain 
subpopulations of African men over others. This would have implications for the general utility of PHS46+African and provide an opportunity to further improve the model.

Therefore, we used principal component (PC) analysis to estimate the genetic relatedness ${ }^{13,14}$ of a dataset of men of African genetic ancestry (African dataset) and to

5 determine whether the performance of PHS46+African varied along PCs representing local genetic ancestry near African-specific SNPs. The PC analysis was conducted on a SNP window encompassing the 3 African-specific SNPs and limited to the 8q24 chromosome. In this way, the African men are projected onto axes, where proximity is based on patterns of genetic variation within this specific section of the genome and agnostic to geographical or social groupings in

10 the dataset. We believe that this "local PC" approach ${ }^{13}$, focusing on 8q24, may uncover subpopulations of African men for whom the utility of PHS46+African differs.

\section{Material and Methods}

African-Ancestry Dataset

Genotypic and phenotypic data for this study were obtained from the Prostate Cancer Association Group to Investigate Cancer Associated Alterations in the Genome (PRACTICAL) ${ }^{15}$ consortium. Genotyping was performed using the OncoArray ${ }^{15,16}$ chip and covered 444,323 SNPs across the genome. Genotypic data was coded as effect allele counts $(0,1$, or 2$)$ for each SNP. Phenotypic data consisted of the prostate cancer case/control status, age at 20 diagnosis, age at last follow-up, genome-wide principal components, and genotypic ancestry. The genotypic ancestry of each individual was previously estimated using 2,318 ancestry informative markers spanning the entire genome ${ }^{17}$.Ancestral groupings using SNP markers showed good agreement with self-reported race/ethnicity ${ }^{9}$. In total, 6,253 men were classified as having African genotypic ancestry and were used for this analysis. All contributing

25 studies were approved by the relevant ethics committees; written informed consent was obtained from the study participants. 
PHS46+African was estimated for each African individual using the 49 SNPs and their respective PHS46+African SNP coefficients from the literature ${ }^{18}$.

\section{Principal components of 8q24 SNP window}

Genetic variation within a narrow window surrounding the three 8q24 SNPs was quantified using principal component decomposition. An initial selection window of SNPs was identified as all SNPs on chromosome 8 lying between the three African-specific 8q24 SNPs in PHS46+African or within $15 \mathrm{kbp}$ of those SNPs in either direction (Figure 1). SNPs were subsequently removed from this window if the call rate was less than 0.95 or if the SNPs could

10 not be cross-referenced against those available on the 1000 Genomes Project Phase 3 dataset $^{19}$. In total, 93 SNPs (Supplementary Table 1) met the selection criteria and constituted the local 8q24 window. Missing SNP calls were replaced with the mean of the genotyped data for that SNP in the African dataset ${ }^{11,20}$. Genetic count data were first standardized across the dataset (mean of 0 , standard deviation of 1 ) before the first 93 principal components (PC) of the

$158 q 24$ SNP window were estimated using the "pca" function in MATLAB.

\section{Interaction between 8q24 SNP window and PHS46+African}

For each principal component (PC) of the 8q24 SNP window, a Cox proportional hazards model was estimated using the age of onset of any prostate cancer as the time-toevent (Eq. 1):

$$
H R=\exp \left[\left(\beta_{1}+\beta_{2} \times P C+\sum_{j=1}^{4} \beta_{j+2} \times \text { globPC }\right) \times P H S 46+\text { African }\right]
$$

where HR is the hazard rate, $\beta_{1}$ is the coefficient of PHS46+African, $\beta_{2}$ is the coefficient of the interaction terms between the 8q24 PC and PHS46+African, and $\beta_{3}$ through $\beta_{6}$ are the coefficients for the interaction terms between the first 4 global ancestry principal components 
(globPC) and PHS46+African. The model was estimated using the entire African dataset, where each observation was weighted by a sample-weighting correction factor ${ }^{11,20}$ to account for differences between the case-control rate of our dataset and that observed in the general population. Controls were censored at the age of last follow-up. The p-value associated with $\beta_{2}$

5 was recorded for each of the 90 PCs from 8q24, after which the two principal components with the smallest $\mathrm{p}$-values $\left(P C_{1}^{\min }, P C_{2}^{\text {min }}\right)$ were selected for further analysis. Only two components were selected in order to simplify the methodology and visualization of the dataset in this first analysis of the complex interactions of local ancestry on polygenic score performance. This twocomponent selection also reflects what has often been used in the literature to estimate global ancestry classifications ${ }^{17}$.

A Cox proportional hazards model was then estimated using both of the selected principal components (Eq. 2):

$$
H R=\exp \left[\left(\gamma_{1}+\gamma_{2} \times P C_{1}^{\text {min }}+\gamma_{3} \times P C_{2}^{\text {min }}+\sum_{j=1}^{4} \gamma_{j+3} \times \text { globPC }\right) \times P H S 46+\text { African }\right]
$$

The calibration factor (CF) for PHS46+African, as a function of $P C_{1}^{\min }$ and $P C_{2}^{\min }$, was defined 15 as (Eq. 3):

$$
C F=\gamma_{1}+\gamma_{2} \times P C_{1}^{\text {min }}+\gamma_{3} \times P C_{2}^{\text {min }}
$$

CF was formulated as the performance metric of interest in this study and can be used to quantify the variation in the coefficient of PHS46+African as a result of differences in the expression of variants within the 8q24 SNP window. Larger values of CF suggest stronger associations between the PHS46+African score and the age at diagnosis of prostate cancer. Individuals in the top $33^{\text {rd }}$, middle $33^{\text {rd }}$, and bottom $33^{\text {rd }}$ percentiles of CF values were grouped into high-, middle- and low-calibration groups respectively. These groups were used to facilitate comparisons in CF between PHS models and dataset variables. 


\section{Comparison between PHS46 and PHS46+African in calibration groups}

Within each calibration group, a Cox proportional hazards model was fit using the age of diagnosis of any prostate cancer as the time-to-event and either PHS46+African or PHS46 as the sole predictor variable. PHS46 was estimated using SNP coefficients published previously ${ }^{18}$.

5 Controls were censored at age at last follow-up. Models were fit using data only from that calibration group. For each of these group-based models, the explained relative risk (ERR) ${ }^{21}$ was estimated as a measure of model goodness-of-fit. ERR was compared between the two PHS models to determine whether the improvement in performance between the models was evenly distributed across the calibration groups. Empirical confidence intervals for ERR were

10 estimated using 1000 bootstrapped samples.

\section{$\underline{\text { Variation in dataset variables across calibration groups }}$}

In order to identify differences in characteristics between calibration groups, a set of generalized linear models were fit to study the association between dataset variables (genetic count of three African-specific 8q24 SNPs, case-control status, and age at diagnosis of cases) and calibration groups. In each case, the dataset variable was set as the independent variable, while the calibration group classification (low/middle/high) was set as the predictor. Identity link functions were used for the continuous dataset variables (genetic count of three 8q24 SNPs, age at diagnosis of cases), whereas the logit link function was used for the binary variable

20 (case-control status). Fitted models were then used to predict mean values of the dataset variables for each 2-PC region. In addition, a chi-squared test was used to determine whether any association existed between the contributing study (18 in total) and calibration group.

\section{$\underline{\text { Continental differences between calibration groups }}$}

The 1000 Genomes (1000G) dataset was used to identify potential differences in continental origins across calibration groups. The $1000 \mathrm{G}$ dataset is subdivided into 5 continental 
groups: European, East Asian, admixed American, South Asian, and African individuals.

Genetic counts for the 93-SNP 8q24 window for 2,504 individuals from the 1000G dataset were obtained from publicly available sources ${ }^{19} . P C_{1}^{\min }$ and $P C_{2}^{\min }$ scores were estimated using the same scaling factors and PC coefficients derived from the African dataset. CF and calibration

5 groups for each individual in the 1000G dataset were estimated using Equation 3, and the percentile cutoffs derived from the African dataset. Chi-squared tests were used to test for associations between continental and calibration groups.

\section{Results}

Model interaction between 8q24 PCs and PHS46+African

The first (PC-1) and twentieth (PC-20) principal components had the two smallest $p$ values (Supplementary Table 2) when estimating the Cox proportional hazards models, as formulated in Eq.1, for all 93 principal components of the 8q24 SNP window. These two principal components were thus selected as $P C_{1}^{\text {min }}$ and $P C_{2}^{\text {min }}$ and used to estimate the Cox

15 proportional hazards model formulated in Eq. 2. (Table 1). No significant interactions $(\alpha=0.05)$ were detected between global ancestry principal components and PHS46+African.

Principal component coefficients and scaling factors needed to estimate PC-1 and PC-20 from the genetic counts of the 93 SNP-8q24 window are reported in the Supplementary Material

20 (Supplementary Table 3).

\section{Calibration factor for PHS46+African}

CF was plotted as a function PC-1 and PC-20 for every African individual in our dataset (Figure 2A). Qualitatively, the calibration factor tended to increase from high-PC-1/low-PC-20 to 25 low-PC-1/high-PC-20 values from a minimum value of 0.41 to a maximum of 2.94 . Grouping the 
African individuals based on percentiles of CF (Figure 2B) demonstrates this pattern more conspicuously, as the middle calibration group clusters on the PC-1/PC-20 space as a diagonal band that neatly divides the high and low calibration groups.

To investigate the influence of limiting the analysis to PC-1 and PC-20, we also

5 estimated a calibration factor using the three principal components with the smallest p-values for $\beta_{2}$ in Equation 1. The resulting 3-PC calibration factor was highly correlated with $\mathrm{CF}\left(\mathrm{R}^{2}=\right.$ 0.83), suggesting that similar trends would be observed if additional local ancestry PC's were incorporated into the formulation of CF.

10 Comparing explained relative risk (ERR) between calibration groups and across PHS models

Mean ERR values for both PHS46 and PHS46+African were greater for the high calibration group than for the low calibration group (Table 2). Improvement of ERR with addition of the three African-specific SNPs was estimated as the as the difference between the ERR values of PHS46+African and PHS46. The absolute improvement in ERR was comparable for

15 all three calibration groups.

\section{Variation in dataset variables across 2-PC space}

Fitted generalized linear models were used to predict mean values of case-fraction, age of cases, and genetic counts of three 8q24 SNPs for the calibration groups (Supplementary

20 Table 4). No significant $(p<0.05)$ differences were found in the predicted age of cases across the groups. The predicted fraction of cases in the high-calibration group was lower (0.49) than that of the low-calibration group (0.53). The predicted mean genetic count of rs5013678 in the high-calibration group (0.27) was greater than that in the low-calibration (0.092) and middlecalibration (0.17) groups. No statistically significant association was detected between

25 contributing study and calibration group (Supplementary Table 5). 
As a post-hoc analysis, $\mathrm{R}^{2}$ values were estimated between case-control status and CF (0.0025) as well as between mean genetic count of rs5013678 and CF (0.039), suggesting that less than $4 \%$ of the variation in CF could be explained by each of these variables.

\section{Continental characterization of calibration groups}

The $1000 \mathrm{G}$ dataset was mapped into the 2-dimensional space defined by PC-1 and PC20 and stratified by continental group (Figure 3). In general, individuals from the $1000 \mathrm{G}$ dataset mapped within the boundaries defined by the OncoArray African dataset. A statistically significant association $\left(\chi^{2}=288, p<0.001\right)$ was detected between continental and calibration groups in the $1000 \mathrm{G}$ dataset. Analysis of the standardized residuals of the test (Supplementary Table 6) revealed that the largest deviation from the expected cross-tabulation counts of the two variables was the greater-than-expected number of African individuals in the low-calibration group. Further analysis of the cross-tabulation between the 2 variables (Supplementary Table 7) revealed that the individuals of European origin made up the largest continental group within the

15 high-calibration individuals (20\%).

\section{Discussion}

Genetic models developed predominantly with European data may widen health disparities. PHS46+African incorporates African-specific SNPs from 8q24 and improves

20 performance in men of African ancestry, but we have demonstrated here that heterogeneity in local ancestry in 8q24 can affect performance gains.

We identified two principal components within the 8q24 region (PC-1 and PC-20) that were most strongly associated with the calibration factor of PHS46+African among men of African ancestry. These associations were estimated while co-varying for global ancestry

25 principal components (Table 1), suggesting that the variation in performance of PHS46+African across men of African ancestry using local ancestry in 8q24 could not be explained by ancestral 
differences estimated using the entire genome. The model-predicted CF values ranged from 0.41 to 2.94 for the combinations of PC-1 and PC-20 found in our dataset, suggesting that PHS46+African was roughly 7 times more useful in stratifying risk in some individuals compared to others. Assigning individuals into three equally sized subsets based on thresholds of CF, we 5 identified low-, middle-, and high-calibration groups within our dataset. The goodness-of-fit for PHS46+African in each of these groups, assessed using the explained relative risk, was also found to increase from low- to high-calibration group. Improvements in ERR were observed across all groups when PHS46+African was used instead of PHS46.

The discovery of PC-1 and PC-20 allows us to identify individuals for whom risk

10 stratification using PHS46+African is expected to be less precise. Several strategies could be implemented to improve equity in performance of PHS46+African in men of African genetic ancestry. These strategies can be pursued simultaneously with essential work to increase the overall diversity of genetic studies. For example, efforts to discover additional SNPs in future datasets could include weighting of individuals according to their PC-1 and PC-20 values, in order to enrich discovery of SNPs that preferentially benefit those in the low-calibration group. In addition, SNP weights in PHS46+African might be re-estimated to account for variations in effect sizes with calibration group. The principal components can also be used prospectively to guide enrollment in genome wide association studies so as to ensure that individuals from lowcalibration groups are adequately represented in the datasets.

By cross-referencing our dataset with individuals from 1000 Genomes, we identified continent-level differences in calibration groups that suggest ancestral origins for the genetic relatedness defined by PC-1 and PC-20. The mapping of 1000G data into the 2-dimensional space defined by PC-1 and PC-20 may provide clues as to the underlying variation in CF. 46 of the 49 SNPs used in PHS46+African were discovered in a dataset consisting entirely of men

25 with European genetic ancestry. Therefore, it is unsurprising that the high-calibration group overlapped substantially with men of European ancestry, as defined by $1000 \mathrm{G}$. However, 
certain continental groups that were not explicitly used in training of model weights, such as South Asian and admixed American, also exhibited overlap with the high-calibration group (Supplementary Table 7). On the opposite end of the spectrum, the low-calibration group overlapped with primarily African and East Asian men. Further investigation into pockets of 5 reference groups, from $1000 \mathrm{G}$ and other datasets, that share common values of PC-1 and PC20 may reveal ancestral linkages that may help to predict which ancestral groups or datasets a model is most likely to perform well in.

There are several limitations to the current analysis to consider. We studied the same dataset that was used to identify the 3 African-specific SNPs. Therefore, the dependence of

10 PHS46+African on PC-1 and PC-20 will need to be validated in independent test sets to ensure generalizability of findings. Our study is further limited by a relatively small number of observations compared to those found in larger, often predominantly European, genome-wide association studies. While a larger dataset would allow us to more robustly estimate model coefficients, the mapping of $1000 \mathrm{G}$ data into the same 2-dimensional space suggests that our

15 current sample accurately portrays the extent of variation in the 8q24 SNP window (Figure 3). In addition, the calibration groups were selected based on arbitrary thresholds of CF and were used solely to simplify the analysis. Further investigations will be required to determine whether distinct subpopulations, based on ancestral relatedness, may exist in the 2-dimensional PC space. Furthermore, 8q24 was chosen to investigate effects in dependence of PHS

20 performance on local ancestry because of substantial evidence supporting the importance of this region of the genome to the prediction of prostate cancer in men of African ancestry. The techniques described in this study can be used, in the future, to investigate local ancestry effects across the genome. Lastly, the dataset used in this analysis may only reflect a fraction of the diversity that is present in men of African genetic ancestry. As such, we are most likely 25 under-estimating the variation in real-world performance of PHS46+African in this population. 
In conclusion, we used local PC analysis to identify axes of variation within the 8q24 SNP window that were strongly associated with the performance of PHS46+African. Mapping our dataset onto these axes revealed that PHS46+African may meaningfully underperform in certain individuals of African genetic ancestry. Investigation into the origins of both high- and

5 low-performing groups can be used to generate a model that is more equitable in performance across subpopulations of men with African ancestry. 


\section{Ethics Statement}

The present analyses used de-identified data from the PRACTICAL consortium and have been approved by the Institutional Review Board at the University of California San Diego. All contributing studies were approved by the relevant ethics committees and performed in

5 accordance with the Declaration of Helsinki. 


\section{Conflict of Interest:}

All authors declare no support from any organization for the submitted work except as follows: AMD and TMS report a research grant from the US Department of Defense. OAA reports research grants from KG Jebsen Stiftelsen, Research Council of Norway, and South East

\section{Norway Health Authority.}

Authors declare no financial relationships with any organizations that might have an interest in the submitted work in the previous three years except as follows, with all of these relationships outside the present study: TMS reports honoraria from Multimodal Imaging Services Corporation for imaging segmentation, honoraria from WebMD, Inc. for educational content, as

10 well as a past research grant from Varian Medical Systems. OAA reports speaker honoraria from Lundbeck.

Authors declare no other relationships or activities that could appear to have influenced the submitted work except as follows: OAA has a patent application \# U.S. 20150356243 pending; AMD also applied for this patent application and assigned it to UC San Diego. AMD has

15 additional disclosures outside the present work: founder, equity holder, and advisory board member for CorTechs Labs, Inc.; founder and equity holder in HealthLytix, Inc., advisory board member of Human Longevity, Inc.; recipient of nonfinancial research support from General Electric Healthcare. OAA is a consultant for HealthLytix, Inc.

Additional acknowledgments for the PRACTICAL consortium and contributing studies

20 are described in the Appendix A3

\section{Funding}

This study was funded in part by grants from the University of California (\#C21CR2060), the United States National Institute of Health/National Institute of Biomedical Imaging and

25 Bioengineering (\#K08EB026503), the Research Council of Norway (\#223273), KG Jebsen Stiftelsen, and South East Norway Health Authority. 
Funding for the PRACTICAL consortium member studies is detailed in the Appendix A2.

The content is solely the responsibility of the authors and does not necessarily represent the official views of any of the funding agencies, who had no role in the design and conduct of the

5 study; collection, management, analysis, and interpretation of the data; preparation, review, or approval of the manuscript; and decision to submit the manuscript for publication. 


\section{Data Availability Statement}

The data used in this work were obtained from the Prostate Cancer Association Group to Investigate Cancer Associated Alterations in the Genome (PRACTICAL) consortium.

Readers who are interested in accessing the data must first submit a proposal to the Data

5 Access Committee. If the reader is not a member of the consortium, their concept form must be sponsored by a principal investigator (PI) of one of the PRACTICAL consortium member studies. If approved by the Data Access Committee, PIs within the consortium, each of whom retains ownership of their data submitted to the consortium, can then choose to participate in the specific proposal. In addition, portions of the data are available for request from dbGaP

10 (database of Genotypes and Phenotypes) which is maintained by the National Center for Biotechnology Information (NCBI):

https://www.ncbi.nlm.nih.gov/gap/?term=lcogs+prostatehttps://www.ncbi.nlm.nih.gov/gap/?term $=$ Icogs + prostate.

Anyone can apply to join the consortium. The eligibility requirements are listed here:

$15 \mathrm{http}: / /$ practical.icr.ac.uk/blog/?page_id=9. Joining the consortium would not guarantee access, as a proposal for access would still be submitted to the Data Access Committee, but there would be no need for a separate member sponsor. Readers may find information about application by using the contact information below:

20 Rosalind Eeles

Principal Investigator for PRACTICAL

Professor of Oncogenetics

Institute of Cancer Research (ICR)

Sutton, UK

25 Email: PRACTICAL@icr.ac.uk

URL: http://practical.icr.ac.uk

Tel: ++44 (0)20 87224094 


\section{References}

1. Seibert TM, Fan CC, Wang Y, Zuber V, Karunamuni R, Parsons JK, Eeles RA, Easton DF, Kote-Jarai Z, Al Olama AA, Garcia SB, Muir K, et al. Polygenic hazard score to guide screening for aggressive prostate cancer: Development and validation in large scale cohorts. BMJ 2018;360:1-7.

2. Huynh-Le M-P, Fan CC, Karunamuni R, Walsh El, Turner EL, Lane JA, Martin RM, Neal DE, Donovan JL, Hamdy FC, Parsons JKK, Eeles RA, et al. A genetic risk score to personalize prostate cancer screening, applied to population data. Cancer Epidemiol Biomarkers Prev 2020;cebp.1527.2019.

10 3. Pashayan N, Duffy SW, Chowdhury S, Dent T, Burton H, Neal DE, Easton DF, Eeles R, Pharoah P. Polygenic susceptibility to prostate and breast cancer: Implications for personalised screening. Br J Cancer [Internet] 2011;104:1656-63. Available from: http://dx.doi.org/10.1038/bjc.2011.118

4. Witte JS. Personalized prostate cancer screening: Improving PSA tests with genomic information. Sci Transl Med 2010;2:1-5.

5. Chen H, Liu X, Brendler CB, Ankerst DP, Leach RJ, Goodman PJ, Lucia MS, Tangen CM, Wang L, Hsu FC, Sun J, Kader AK, et al. Adding genetic risk score to family history identifies twice as many high-risk men for prostate cancer: Results from the prostate cancer prevention trial. Prostate 2016;76:1120-9.

20 6. Duncan L, Shen H, Gelaye B, Meijsen J, Ressler K, Feldman M, Peterson R, Domingue B. Analysis of polygenic risk score usage and performance in diverse human populations. Nat Commun [Internet] 2019;10. Available from: http://dx.doi.org/10.1038/s41467-019$11112-0$

7. Sirugo G, Williams SM, Tishkoff SA. The Missing Diversity in Human Genetic Studies. Cell [Internet] 2019;177:26-31. Available from: https://doi.org/10.1016/j.cell.2019.02.048

8. DeSantis CE, Miller KD, Goding Sauer A, Jemal A, Siegel RL. Cancer statistics for 
African Americans, 2019. CA Cancer J Clin 2019;69:211-33.

9. Huynh-Le M-P, Fan CC, Karunamuni R, Thompson WK, Martinez ME, Eeles RA, KoteJarai Z, Muir K, Collaborators U, Schleutker J, Pashayan N, Batra J, et al. Polygenic hazard score is associated with prostate cancer in multi-ethnic populations. medRxiv 2020;1-34.

10. Martin AR, Kanai M, Kamatani Y, Okada Y, Neale BM, Daly MJ. Clinical use of current polygenic risk scores may exacerbate health disparities. Nat Genet [Internet] 2019;51:584-91. Available from: http://dx.doi.org/10.1038/s41588-019-0379-x

11. Karunamuni RA, Huynh-Le M-P, Fan CC, Thompson W, Eeles RA, Kote-Jarai Z, Muir K, Lophatananon A, Tangen CM, Goodman PJ, Thompson IMJ, Blot WJ, et al. Africanspecific improvement of a polygenic hazard score for age at diagnosis of prostate cancer. Int J cancer 2020;

12. Mathias RA, Taub MA, Gignoux CR, Fu W, Musharoff S, O'Connor TD, Vergara C, Torgerson DG, Pino-Yanes M, Shringarpure SS, Huang L, Rafaels N, et al. A continuum of admixture in the Western Hemisphere revealed by the African Diaspora genome. Nat Commun 2016;7.

13. Li H, Ralph P. Local PCA Shows How the Effect of Population. Genetics [Internet] 2019;211:289-304. Available from:

https://search.proquest.com/docview/2168065525/fulltextPDF/40FB8A65E6B34C81PQ/1 ?accountid $=12598$

14. Todesco M, Owens GL, Bercovich N, Légaré JS, Soudi S, Burge DO, Huang K, Ostevik KL, Drummond EBM, Imerovski I, Lande K, Pascual-Robles MA, et al. Massive haplotypes underlie ecotypic differentiation in sunflowers. Nature [Internet] 2020;584:602-7. Available from: http://dx.doi.org/10.1038/s41586-020-2467-6

25 15. Amos Cl, Dennis J, Wang Z, Byun J, Schumacher FR, Gayther SA, Casey G, Hunter DJ, Sellers TA, Gruber SB, Dunning AM, Michailidou K, et al. The oncoarray consortium: A 
network for understanding the genetic architecture of common cancers. Cancer Epidemiol Biomarkers Prev 2017;26:126-35.

16. Infinium OncoArray-500K BeadChip | Research cancer predisposition and risk [Internet]. [cited 2020 Jul 21];Available from: https://www.illumina.com/products/by-type/microarraykits/infinium-oncoarray-500k.html

17. Li Y, Byun J, Cai G, Xiao X, Han Y, Cornelis O, Dinulos JE, Dennis J, Easton D, Gorlov I, Seldin MF, Amos Cl. FastPop: A rapid principal component derived method to infer intercontinental ancestry using genetic data. BMC Bioinformatics [Internet] 2016;17:1-8. Available from: http://dx.doi.org/10.1186/s12859-016-0965-1

10 18. Huynh-Le M-P, Chieh Fan C, Karunamuni R, Martinez ME, Eeles RA, Kote-Jarai Z, Muir K, Collaborators U, Schleutker J, Pashayan N, Batra J, APCB (Australian Prostate Cancer Bioresource), et al. Polygenic hazard score is associated with prostate cancer in multi-ethnic populations. medRxiv 2019;

19. Auton A, Abecasis GR, Altshuler DM, Durbin RM, Bentley DR, Chakravarti A, Clark AG, Donnelly P, Eichler EE, Flicek P, Gabriel SB, Gibbs RA, et al. A global reference for human genetic variation. Nature 2015;526:68-74.

20. Karunamuni RA, Huynh-Le MP, Fan CC, Eeles RA, Easton DF, Kote-Jarai ZsS, Amin Al Olama A, Benlloch Garcia S, Muir K, Gronberg H, Wiklund F, Aly M, et al. The effect of sample size on polygenic hazard models for prostate cancer. Eur J Hum Genet 2020;

20 21. Heller G. A measure of explained risk in the proportional hazards model. Biostatistics 2012;13:315-25. 


\section{Figure Legends}

Figure 1. Definition of $\mathbf{8 q 2 4}$ SNP window. A window spanning roughly $15 \mathrm{kbp}$ in either direction from the three 8q24 SNPs (rs76229939, rs74421890, rs5013678) was defined as the $5 \quad 8 q 24$ SNP window.

Figure 2. A. CF as a function of PC-1 and PC-20. Calibration factor (CF, Eq. 3) plotted as a function of PC-1 and PC-20. Each point represents an African individual. The calibration factor tended to increase from high-PC-1/low-PC-20 (bottom right corner) to low-PC-1/high-PC-20 (top left corner) values. B. Calibration groups as a function of PC-1 and PC-20. Individuals from

10 the African dataset classified into low-(red points, minimum to $33^{\text {rd }}$ percentile), middle-(green points, $33^{\text {rd }}$ to $67^{\text {th }}$ percentile), and high-(blue points, $67^{\text {th }}$ to maximum) calibration groups. Figure 3. 1000G dataset mapped to PC-1 and PC-20. 2,504 individuals from the $1000 \mathrm{G}$ dataset (purple dots) were mapped onto the 2-dimensional space defined by PC-1 and PC-20. Each pane represents a different continental group: African, admixed American, East Asian,

15 European, and South Asian. The mapping is overlaid on a grayscale version of Figure 2B (i.e., gray dots represent individuals in the African-Ancestry Dataset used in the present study). 
Table 1. Coefficients of Cox model estimating interactions between PC and

PHS46+African. Estimates of coefficients from the Cox proportional hazards model describing the association between age of onset of any prostate cancer as the time-to-event, and interactions between 8q24 PCs (PC-1, PC-20) and PHS46+African as the predictors. The model

5 also included interaction terms between the first 4 global ancestry PCs and PHS46+African as covariables.

\begin{tabular}{cccc}
\hline Coefficient & Associated predictor & Estimate & p-value \\
\hline$\gamma_{1}$ & PHS46+African & 1.55 & $<1 \mathrm{E}-16$ \\
$\gamma_{2}$ & PC-1 x PHS46+African & -0.049 & $7.36 \mathrm{E}-6$ \\
$\gamma_{3}$ & PC-20 x PHS46+African & 0.21 & $5.84 \mathrm{E}-5$ \\
$\gamma_{4}$ & Global Ancestry PC(1-4) x & -14.71 & 0.29 \\
$\gamma_{5}$ & PHS46+African & -3.97 & 0.33 \\
$\gamma_{6}$ & & -2.86 & 0.42 \\
$\gamma_{7}$ & & & 0.54 \\
\hline
\end{tabular}


Table 2. ERR for PHS46+African and PHS46. Mean explained relative risk (ERR) values were tabulated for each of calibration groups using either PHS46 or PHS46+African models. No statistically significant differences in absolute improvement in ERR were observed for any of the calibration groups. Values are tabulated as mean [95\% confidence interval].

5

\begin{tabular}{cccc}
\hline $\begin{array}{c}\text { Calibration } \\
\text { group }\end{array}$ & PHS46+African & PHS46 & Improvement in ERR \\
\hline low & $0.036[0.019,0.059]$ & $0.013[0.004,0.028]$ & $0.023[0.011,0.039]$ \\
middle & $0.056[0.036,0.083]$ & $0.018[0.007,0.036]$ & $0.037[0.021,0.055]$ \\
high & $0.099[0.068,0.135]$ & $0.041[0.022,0.069]$ & $0.058[0.035,0.083]$ \\
\hline
\end{tabular}

